\title{
Research on Medical Waste Supervision Model and Implementation Method Based on Blockchain
}

\author{
Hui Wang, ${ }^{1}$ Longshuai Zheng, ${ }^{2}$ Qiuhong Xue, ${ }^{2}$ and Xueqing Li $\mathbb{D}^{1}$ \\ ${ }^{1}$ School of Software, Shandong University, Jinan 250101, Shandong, China \\ ${ }^{2}$ Institute of Software, Chinese Academy of Sciences, Beijing 100190, China \\ Correspondence should be addressed to Xueqing Li; xqli@sdu.edu.cn
}

Received 9 September 2021; Revised 6 January 2022; Accepted 10 January 2022; Published 24 February 2022

Academic Editor: Lu Liu

Copyright (c) 2022 Hui Wang et al. This is an open access article distributed under the Creative Commons Attribution License, which permits unrestricted use, distribution, and reproduction in any medium, provided the original work is properly cited.

The Internet of Things (IoT) has brought unprecedented changes to the society and permeated our daily life. However, it has not been successfully applied in the area of medical waste regulation, where the recycling and disposal of medical waste have significant loopholes in the management of classification, transportation, disposal, supervision, and other links. The source, authenticity, and integrity of medical waste data lack guarantees, and there is a risk of data tampering and forgery. Although there are currently some medical waste supervision applications combined with IoT-based blockchain domestically and internationally to facilitate information sharing and transfer, no verifiable method is provided for the information privacy leakage of medical waste operators. To address this problem, we propose a blockchain-based medical waste supervision model, which connects participants involved in the process, introduces digital credentials to achieve the protection of operator information privacy, and ensures that the entire data process is authentic and credible. By building a decentralized system architecture and setting intelligent contracts, we integrate and record the medical waste disposal regulatory information in different phases on the blockchain to form the supervision of medical waste chain. In addition, we digitize the physical credentials and certificates using digital credentials to achieve cryptography security and privacy protection. The regulatory model designed in this paper can provide digital certificates of disposal tracking information to the health, environmental protection, and other administrative departments in China. It can provide authoritative evidence for the supervision and accountability of medical waste disposal and support the construction of a new generation of medical waste regulatory information systems in China.

\section{Introduction}

Medical waste, known as "No. 1 hazardous waste," refers to the waste containing directly or indirectly infectious, viral, and other hazards produced by medical and health institutions in medical treatment, prevention, healthcare, and other related activities [1]. China has banned the sale of medical waste for many years. However, driven by interests, individuals still make infusion tubes into plastic raw materials and even make illegal profits by reselling placentas. There are large loopholes in the management of classification, transportation, disposal, and supervision of medical waste recycling and disposal [2]. For example, when the waste handover is recorded manually, the query and statistics work are complicated. It is difficult for managers to monitor this work in real time, and it is difficult to trace the responsibility subject.

To manage medical waste more safely, in July 2018, the National Health Commission launched the pilot application construction project of the provincial credit information management platform and selected Fujian Province and other pilot provinces to build a medical waste supervision system based on IoT-enabled blockchain technology. In 2020, Shandong, Shaanxi, and other provinces were added to continue to expand the scale of this application. With the outbreak of COVID-19, the National Health Commission has increasingly stringent regulatory requirements for medical waste, and new solutions are urgently needed to improve the efficiency of supervision. 
The informatization construction, also known as IoT technology application, helps to improve the standardization in the whole medical waste recycling and disposal [3] process. The application of IoT has made an apparent shift and contributed to operation optimization for all industries, including agriculture [4] and transportation [5]. RFID-enabled or Internet-based devices are connected and organized as an information network, making it efficient and productive to integrate trackable data. IoT has the advantages of real-time performance and all-in-one efficiency that enable users to manage and supervise physically isolated devices as a system.

However, such information-based transformation schemes generally transfer the offline data to online through intelligent devices such as code scanners [4]. This transformation scheme is convenient for the query and statistical analysis of relevant data, but there is no innovation in the management mode and management manners of medical waste. There are still some issues in medical waste recovery and disposal, such as shortage of weight, black-box operation, and tampering with credentials.

Furthermore, integrating IoT with blockchain technology enhances the transparency and credibility of the management process. Blockchain is a distributed ledger technology that combines distributed data storage, point-topoint transmission, consensus, and encryption algorithms [5-9]. Encryption algorithm, blockchain structure, and alliance consensus [10] ensure the authenticity, integrity, and nontampering with data on the chain. Integrating blockchain into the management of the whole process of medical waste recovery and disposal can reduce the risk of data tampering and falsification $[11,12]$, guarantee the safety of medical waste data, and improve the supervision level of medical waste.

Current research on medical waste management using IoT and blockchain focuses on the following aspects. (1) Store the business credentials of each link of recovery and disposal on the blockchain to ensure that the data cannot be tampered with [13]. (2) Unified medical waste management, transport vehicles, and people are realized through joint IoT technology [14, 15]. Verify the validity of clinical waste recovery and disposal process through smart contract [16]. (3) Introduce the token reward and punishment mechanism to force participating entities to comply with medical waste disposal rules and other aspects [17]. However, there is a lack of lifecycle management of medical waste, construction of the alliance of stakeholders, and privacy protection mechanism of the participants in the core link.

In terms of stakeholder decentralized collaboration and privacy protection, the proposed general solution, whether in academia or industry, has been one of the issues focused on for a long time. Once a broad and effective solution can be designed under a reasonable security assumption, its impact is obvious. The technologies that are expected to be close to general solutions currently mainly include secure multiparty calculations based on computational difficulty theory, homomorphic ciphertext calculations, and zero-knowledge proofs. However, the above three types of technologies have significant practical limitations. The industry has also tried to rely on a trusted hardware execution environment to build general solutions, but the effectiveness of its actual privacy protection is difficult to verify publicly.

Blockchain technology is not only a decentralized collaboration solution, but as an effective privacy protection solution, it is promoted by introducing breakthrough optimization factors such as sociology, psychology, and economics principles; rational participant models; and multiparty incentive mechanisms. The balance between insensitive user experience and effective privacy protection approaches Pareto optimality. In the case of medical waste management, blockchain systems can provide medical waste management platforms for the collection of waste in cities to maximize sustainability level in terms of health, social, environmental, and financial aspects. The data and transactions stored on the blockchain are accessible to the stakeholders involved in the forward supply chain and waste management processes through distributed public or private ledgers. The decentralization feature of blockchain increases the trust among stakeholders as it eliminates the need to assess the trustworthiness of the participants.

In this paper, we design a medical waste supervision model based on IoT and blockchain techniques to address the above problems. This model integrates blockchain technology into the generation, transportation, treatment, and destruction of medical waste. By connecting different stages of waste disposal, defining the critical node data on the blockchain, and mutually verifying the data under the blockchain, the security and immobility of the data are ensured while achieving system efficiency. In addition, digital credentials are used to enable users to disclose information to protect the privacy of handover personnel selectively. Moreover, on-net monitoring of the flow of medical waste data allows assessing and detecting medical waste trading violations.

1.1. The Main Contributions of This Article. Compared with the traditional medical waste supervision methods, our proposed IoT and blockchain-based medical waste supervision model has the following advantages:

(1) Data security: the critical data of the medical waste treatment process is linked to a certificate to ensure authenticity and nontampering.

(2) Multiparty participation: the decentralized architecture allows the application scenario of multirole subjects by compartmentalizing the corresponding mission, while taking into account both efficiency and security.

(3) Clear rights and responsibilities: relevant responsible persons and operators need to sign when submitting data to the blockchain, and the time, place, person, operation process, and result of data collection are linked at the same time.

(4) Privacy protection: the IoT operator's private information is hidden through the digital credential, and a unique identifier is given on the blockchain to represent each operator; the factual personnel 
information is stored in the digital certificate, and all parties can verify its authenticity in the process of circulation to prevent privacy disclosure.

In terms of the application and practical value of this model, we developed a medical waste supervision system based on the regulatory model proposed in this paper, the RepChain blockchain essential components $[18,19]$ independently developed by the Institute of Software of the Chinese Academy of Sciences, and related technologies of the IoT [20]. The system has been applied in hospitals, transportation, environmental protection, and disposal institutions in Shandong, Fujian, and other places in China. It can improve management methods, effectively reduce regulatory costs, improve regulatory efficiency, and achieve good results.

1.2. The Organizational Structure of This Article. Section 2 introduces related works and discusses the traceability or supervision of medical waste and other wastes utilizing IoTbased blockchain technology. Section 3 proposes the medical waste supervision model based on reproducing the traditional medical waste management process, including the digital evidence and the blockchain deployment model. Section 4 introduces the medical waste supervision system. Section 5 combines the current situation of the medical waste industry in Shaanxi Province, develops the relevant condition of the medical waste supervision system according to this model, and evaluates the outcome. Section 6 summarizes the paper.

\section{Related Works}

2.1. Background. Technology-driven methods for medical waste management generally use bar codes, two-dimensional codes, RFID, and other [21, 22] IoT-related methods to track and realize the traceability of the whole process of medical waste management. However, they are all based on forming a strictly closed loop in the collection and transportation process. In reality, medical institutions, transport agencies, and disposal agencies have their waste information management platform. The information management systems among medical institutions are also uneven, so it is difficult for the regulatory authorities to carry out unified and effective management. Blockchain-based medical waste management aims to connect these separated systems and create a traceable and transparent, automated rule engine to solve openness, interoperability, and decentralization of medical waste.

2.2. The Research Status at Home and Abroad. In the research of "blockchain + medical waste disposal," Li et al. proposed the integrated development of blockchain and medical waste management. Medical waste disposal operators of each process store relevant information on the blockchain on time. They rely on the tracing source code as the carrier of information transmission, through the tracing source code collection and monitoring data, and tracking and confirming the treatment, transportation, disposal, and other links of medical waste to achieve multidimensional network supervision. In terms of alliance building, it is proposed that hospitals, cleaning companies, the public, and national supervisory agencies participate in the decentralized autonomous organization simultaneously and use the interests of all parties to play a checking role [23]. Ahmad et al. [24] proposed integrating Ethereum and interplanetary file system (IPFS) for the supply chain for COVID-19 medical devices to securely access, store, and share data related to COVID-19 medical devices and their waste management. They also define rules of interaction for waste disposal so that governments can impose penalties on stakeholders if violations occur. Kassou et al. [1] show that medical wastewater is based on blockchain technology and the IoT system to effectively manage, coordinate, and monitor medical wastewater, such as in-hospital using flow meter, water meter, and intelligent Internet of sensing equipment. It will produce the data using blockchain for the witness, to government agencies and stakeholders involved in the common node, which improves the process brightness and supervision.

In patent research and development, Jiang and Tian [25] proposed a blockchain-based medical waste supervision platform and management method. They built a blockchainbased medical waste whole-lifecycle management method in medical institutions through the comprehensive application of blockchain, big data, and IoT technologies to eliminate regulatory gaps and blind areas. Their way solves the longstanding problems of medical institutions, health commissions, and environmental protection departments that cannot be traced, collected, and held accountable. Lin [26] proposed to connect the remote central regulatory server with the device for local treatment of medical waste, the intelligent medical waste collection vehicle, the legal person classification collection bag of the medical department, and the collection and transfer device for disposal of medical waste. Then, the IoT and blockchain were used to achieve intelligent supervision of the whole process, to achieve hierarchical regulation and hierarchical statistical check.

There are few studies on the application of blockchain in the field of medical waste. In terms of sorting out appropriate research methods, it is extended to the area of "blockchain $+\mathrm{e}-$ waste or solid waste" management. Gupta and Bedi [27] proposed an e-waste management system based on Ethereum, which considers the main stakeholders, including electronic component manufacturers, consumers, and retailers, to ensure that participants comply with the e-waste disposal guidelines. According to the defined abnormal events, it uses blockchain to record, report, and verify all electronic waste sold and uses smart contracts to impose penalties on those who are responsible. Laouar et al. [28] proposed the continuous monitoring and tracking of municipal solid waste transportation participants, responsible persons, transportation tracks, collectors, processors, etc. They stored the QR (quick response) codes generated by the data for solid waste identification on the blockchain. Solid waste vehicles' state, location, and routing information are managed using an off-chain storage system to balance safety and throughput. 
2.3. Research Focus of This Article. To sum up, the current IoT-based and blockchain-based medical waste management related studies focus on the following aspects. (1) Save the business credentials of each link of recovery and disposal on the blockchain to ensure data security. (2) Unified medical waste management, transport vehicles, and people are realized through joint IoT. (3) Verify the validity of the clinical waste recovery and disposal process through smart contracts. The current research lacks a mechanism for the whole-lifecycle management of medical waste and the privacy protection of participants in the core link. In contrast, this paper proposes a comprehensive medical waste regulatory model, which combines the domestic medical waste management process with IoT and blockchain technologies to build a multirole entity alliance. In the case of the wholeprocess tracking of the responsible subject, the privacy and security of operators are protected. The result is effective regulation of medical waste in a safe, transparent, trusted, decentralized, and auditable manner.

\section{Medical Waste Supervision Model Based on Blockchain}

In this section, the proposed model for IoT-based and blockchain-based medical waste supervision is introduced. The goal of our system is to ensure transparent and explicit assignment of responsibilities and credible information management for flexible regulation. We demonstrate the process of waste disposal and how we integrate mentioned techniques to guarantee credibility and efficiency. Digital credential is used to assure security and verify authenticity.

3.1. Medical Waste Disposal Process. Based on the Product Lifecycle Management (PLM) [29] model of medical waste and the basic logic of IoT, the whole process of medical waste disposal is supervised from the collection, storage, and transshipment to the disposal and combined with the credit mechanism of blockchain technology and the characteristics of decentralization. A tamper-proof tracking account book for the whole process of medical waste disposal is established to provide users with credible and traceable management information.

This model provides a convenient way for waste disposal personnel to report the data of disposal nodes through smart devices, while providing an aggregated information platform for supervisors to realize the automatic supervision of information by displaying each node of medical waste disposal and monitoring the nodes to promote the timely delivery of medical waste. This process of information collection and supervision improves the standardized management of medical waste disposal, accounts for the medical waste disposal information, and provides a reliable and traceable digital certificate for the supervision and punishment of medical waste disposal. In the disposal process of medical waste, all transaction information occurring on the node is recorded through the contract, so that the process is highly transparent. The proposed IoT blockchain model is shown in Figure 1.

We distribute the responsibilities of different stages in the waste monitor recycle network where timely and credible data exchange plays an essential role. The material and information flow of medical waste treatment is as follows.

3.1.1. Medical Institutions. Medical personnel classify, collect, and sort medical waste according to the Medical Waste Classification Catalog. Medical waste management professionals transport the classified and packaged clinical waste from the medical waste generation site to the temporary storage room in the hospital according to the specified route on a daily basis. The loss and leakage of medical waste should be prevented during transportation. Medical waste management professionals weigh medical waste at the site where it is generated on a daily basis. Registration includes the source, type, weight, delivery time, final destination, and operator. After the medical waste is transferred out, full-time personnel shall clean and disinfect the temporary storage sites and social facilities in a timely manner and make good records.

3.1.2. Transport Company. When collecting clinical waste, the transport personnel of the transport company should register the waste and sign their names. The content of the registration includes the place where clinical waste is generated, the date, the type of waste, and the matters to be explained. The registration transfer form is kept on file at the Medical Waste Management Office for three years. When transporting medical waste, it is necessary to prevent the damage of medical waste containers; the loss, leakage, and diffusion of medical waste; and the direct contact of medical waste with the body. All medical waste should be transported in bags and sealed. Crossing medical areas, human activity areas, food processing areas, etc. should be avoided.

3.1.3. Disposal Company. The medical waste must be disposed of by the centralized medical waste disposal unit approved by the ecological environment department. The whole medical waste treatment process can be traced throughout. Different types of medical waste are absorbed in different ways. For example, the treatment of infectious waste is high temperature incineration. The hazardous waste will be incinerated at high temperatures to destroy its shape and tested for toxicity before being buried.

In the medical waste disposal process (see Figure 2), all transaction information that occurs on the node is recorded through the contract, making the process highly transparent:

(1) The nursing staff and the department staff weigh and encapsulate the medical waste face to face; the nursing staff prints the label, and the department staff scans and confirms the label and transfers the data to the chain.

(2) The nursing staff transports the medical waste to the temporary storage point and conducts face-to-face 


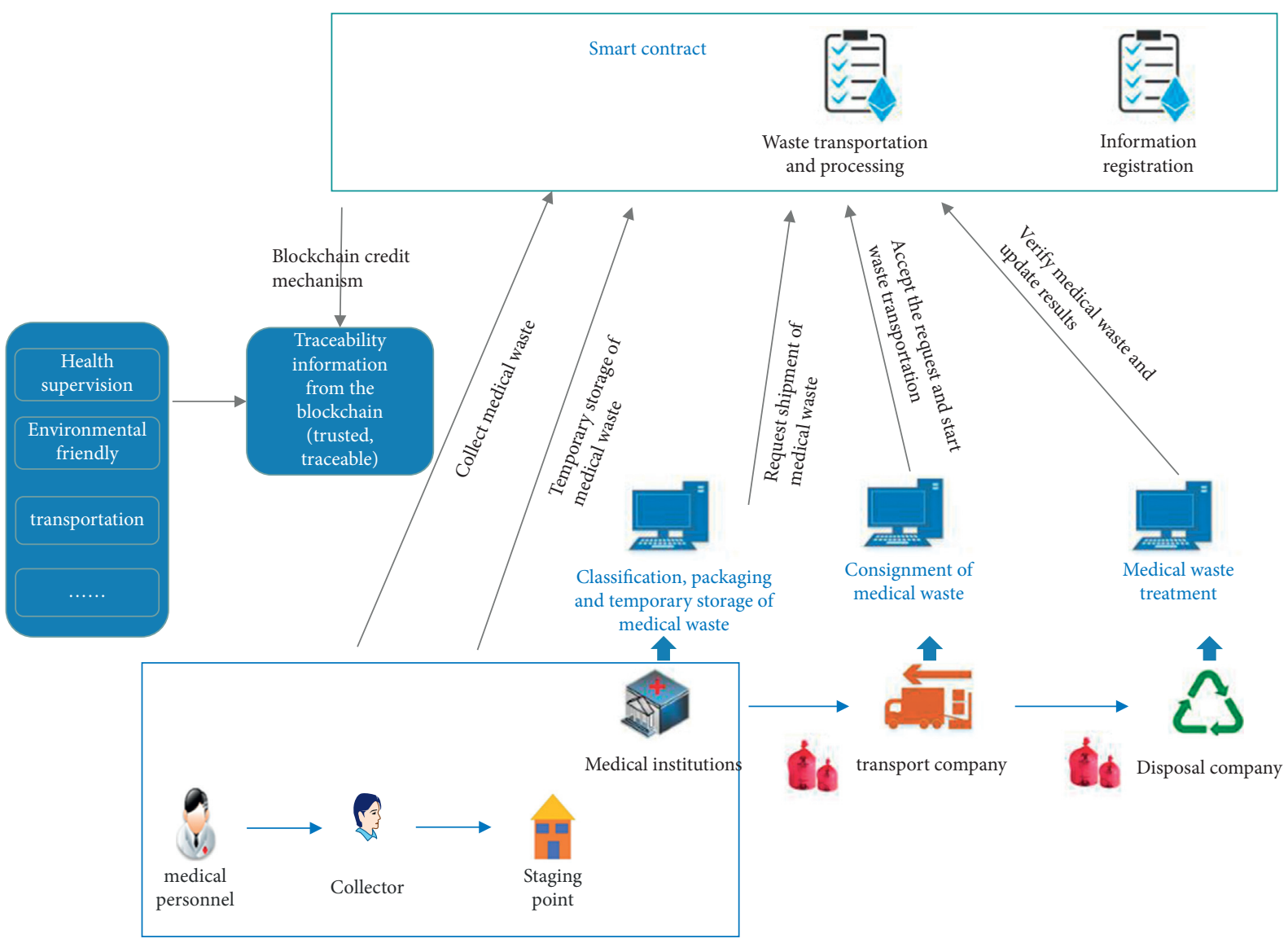

FIgURE 1: The business model of medical waste treatment.

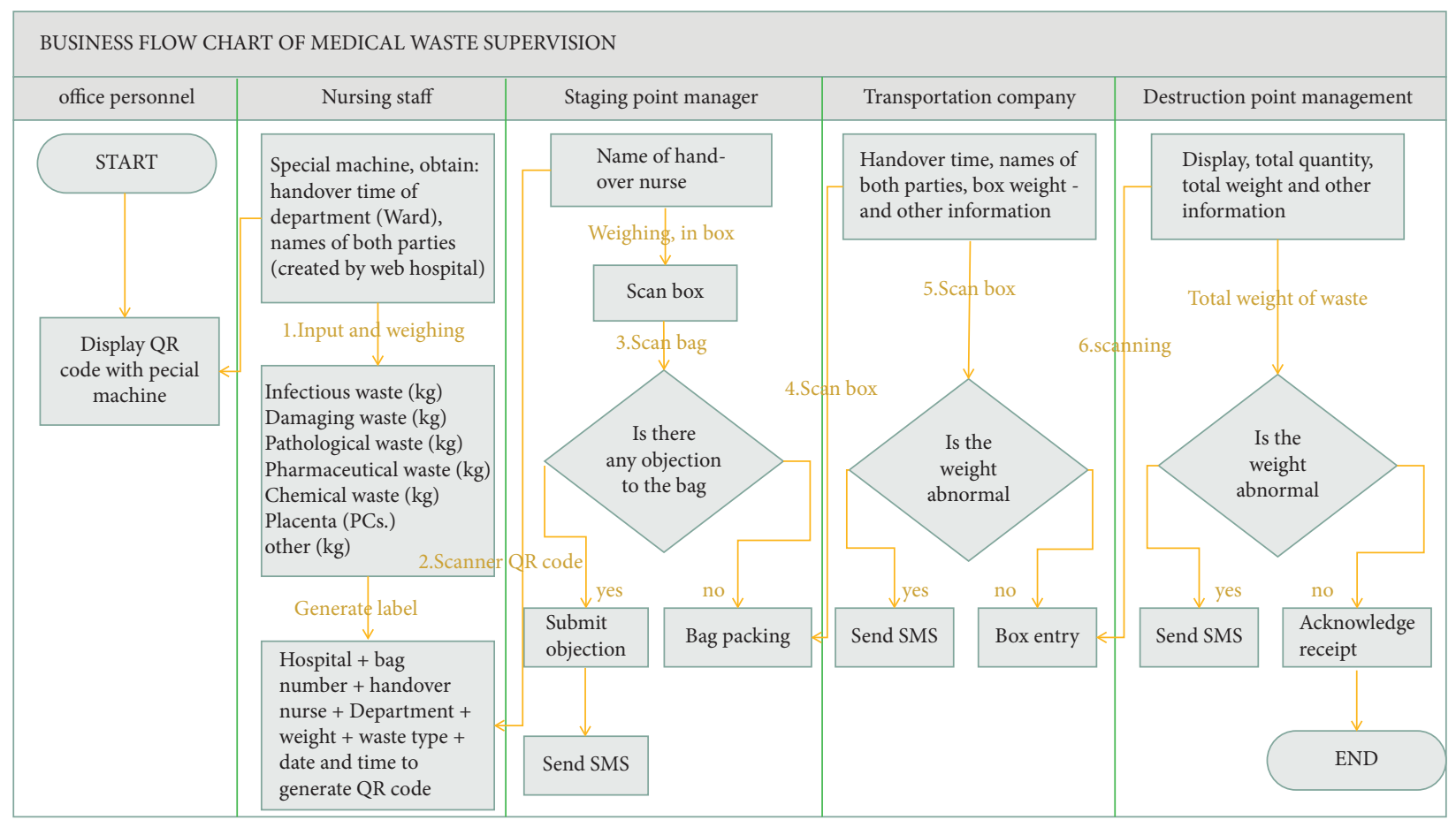

Figure 2: Business flow chart of medical waste supervision. 
weighing and handing over with the temporary storage point management personnel. After confirming that the weight of the waste is correct, a QR code is generated for the box, and the handover data is uploaded to the chain

(3) The management personnel of the hospital's temporary storage point carry out the operation of waste classification and scanning into the bin.

(4) The transportation personnel and the hospital temporary storage point management personnel carry out the weighing and transfer of the boxes. After scanning and confirming that the box weight is correct, the transfer data is uploaded to the chain.

(5) The transportation personnel and the personnel at the destruction point weigh the vehicle, and after confirming the receipt, the handover data will be uploaded to the chain.

\subsection{Digital Credential Model}

Definition 1. A digital credential is a document that is digitized and is cryptographically verifiable, presented in a digital form for easy storage and transmission and based on cryptographic mechanisms to more securely and reliably verify its authenticity.

The digital credential model mainly includes the following:

(i) The certificate issuer, i.e., the medical waste regulatory agency, constructs the digital certificate according to the attribute structure of the digital certificate and is responsible for issuing and updating the certificate.

(ii) The holder of the credential, i.e., the medical waste operator, applies for the digitization of the credential and verifies its correctness.

(iii) Credential verifiers, i.e., participants of medical waste, verify the authenticity and validity of the received digital credentials. Participants include health regulatory authorities, medical institutions, transportation departments, transportation companies, environmental protection departments, and disposal companies.

(iv) Digital credential status includes the following types: valid, revoked, and deactivated.

The digital credential model is illustrated in Figure 3, and an example is shown in Figure 4.

There are many links in the supervision of medical waste, and the privacy disclosure of operators in each link is an essential reason for the relevant personnel to operate in a dark box or engage in dark transactions. To this end, this paper proposes a digital credential model, which hides the operator's private information in the chain, represents each operator by a unique identifier, and stores the basic information in the digital credential.

Operators in all stages of medical waste disposal apply for digital certificates to the regulatory agency. The regulatory agency issues digital credentials with a unique ID (identification number, identity) and signs the digital credential information based on the asymmetric key's digital signature algorithm. After digitizing the credential, the unique ID of the credential, credential status, and other information are uploaded to the chain and available for information verification by other institutions. The specific information of the operator is not disclosed on the chain (or expressed in the form of a pseudonym) to achieve the purpose of protecting the privacy of personnel information.

\subsubsection{Detailed Operation Process}

(1) Applying for digital credentials: medical waste operators need to apply for digital credentials and provide their personal information to the regulatory agency before processing medical waste. The digital credentials are used as a personal identification.

(2) Issuing digital credentials: the regulatory agency obtains the format and structural attributes of the digital credentials that need to be issued from the blockchain, fills in the corresponding information of the operator according to the format and structural attributes, and signs the credential information.

(3) Presenting digital credentials: when the operator accesses the services provided by each participant, each participant informs the operator of the credential information that needs to be provided; the operator finds the credential information that meets the needs of each participant from the digital credentials held by the individual, and provides the credential information to each participant for verification.

(4) Verification of digital credentials: after receiving the credential information of the operator, each participant first verifies the credential information and verifies whether the signature of the presented information is correct; then verifies the correctness of the credential information; and finally obtains it from the blockchain. The validity status of its credentials and corresponding attributes is to determine whether the credentials and corresponding attributes are valid and then determine whether the credential signature information is correct.

(5) Maintaining status: when a regulatory agency issues a credential, it needs to add its credential status information to the blockchain and initialize it; when it needs to change the status of the credential, such as freezing, restoring, or revoking the credential, it needs to change the corresponding credential.

(6) Updating attributes: regulators can update certain attribute values of the issued credentials as needed, without revoking the entire credentials, just reissuing the credentials after the update.

An example of the attribute structure information of a digital credential is shown in Figure 5. 


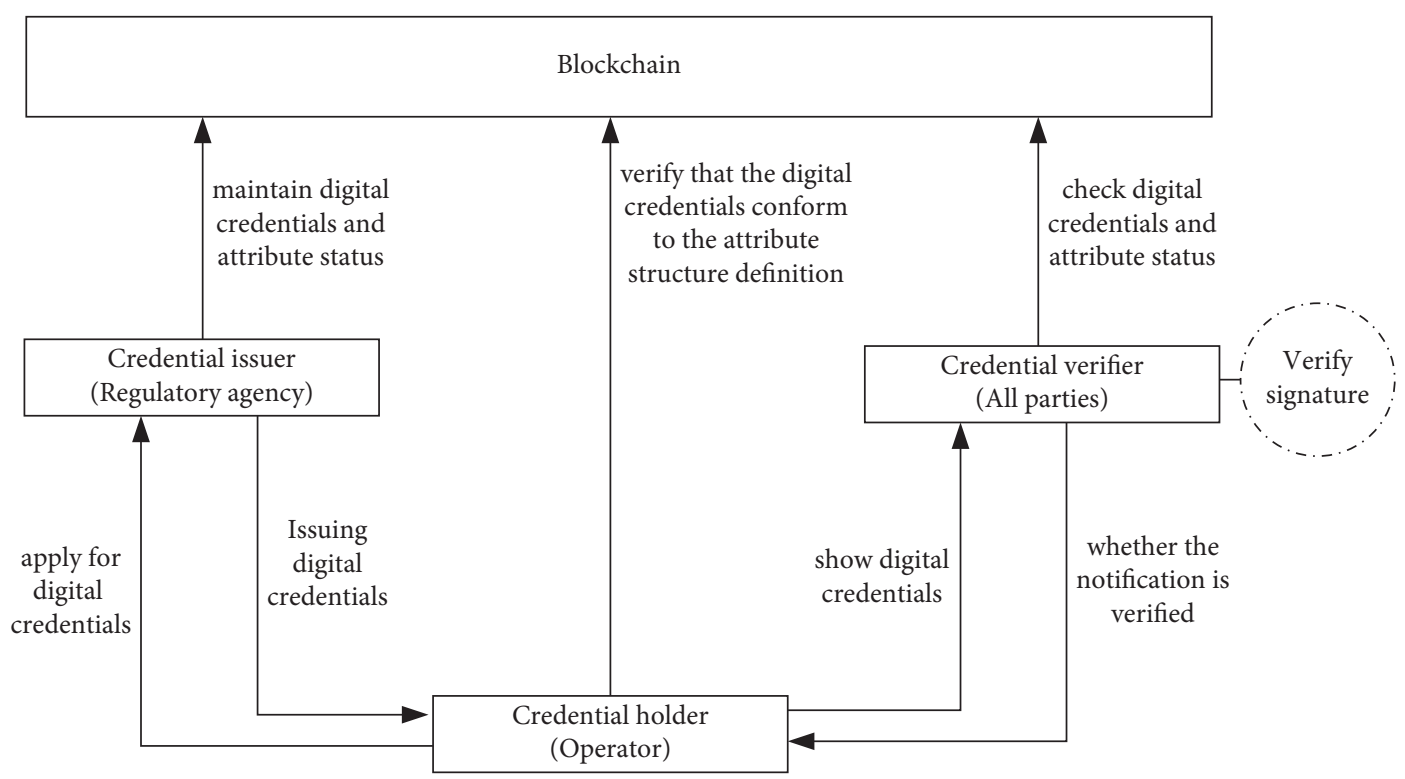

FIgURe 3: Digital credential model.

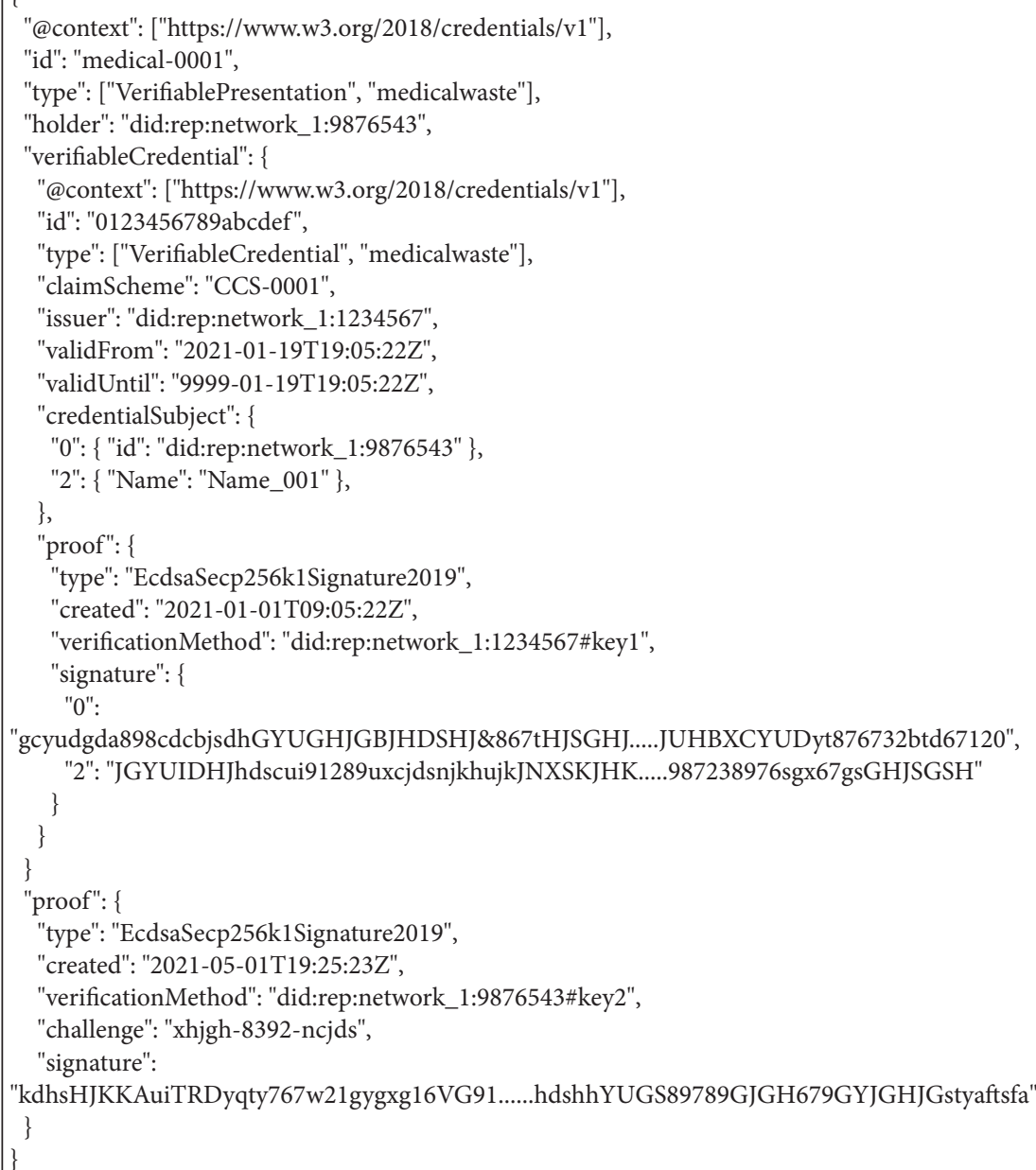

FIGURE 4: Example of digital credential presentation. 


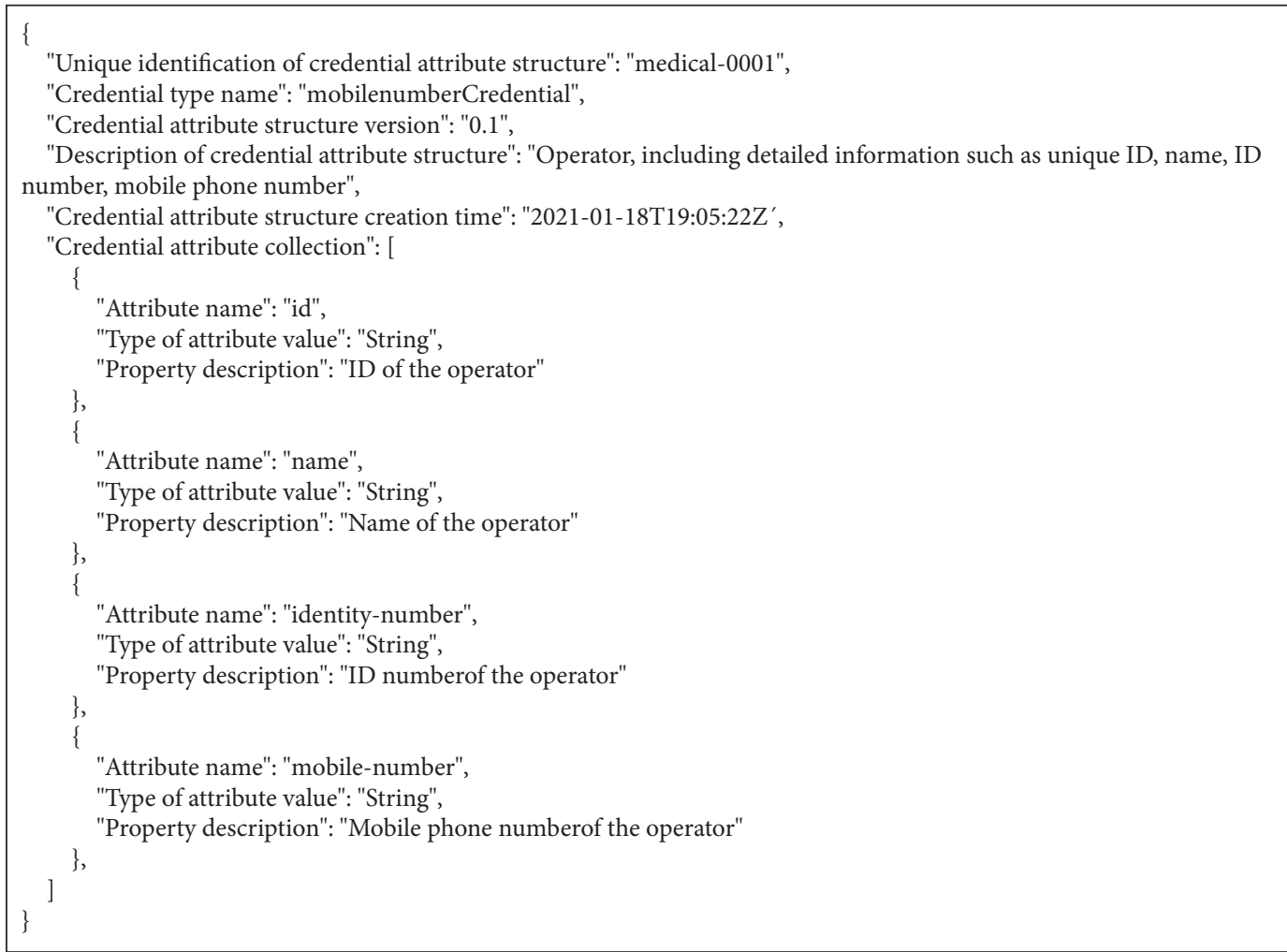

FIGURE 5: Sample diagram of attribute structure information of a digital credential.

\section{Implementation Method of Medical Waste Supervision System Based on Blockchain}

This section presents a detailed analysis of the supervision system built based on the concept of real time and security. We use a clear layered structure and apply RepChain as the evidence chain connecting related practitioners and organizations. The specific implementation method is described as follows.

\subsection{Architecture of Medical Waste Supervision Blockchain} System. The medical waste supervision framework comprises the basic layer of the alliance chain platform and the application layer of medical waste supervision. The former provides IoT-based blockchain services, and the latter provides supervision services for medical waste. From the bottom to the upper layer, there are the data storage, component, application interface, and medical waste supervision application layers, which guarantees the data transfer, as shown in Figure 6.

(1) Data storage layer: it is divided into file storage and database storage, wherein the former mainly stores block segmented files and the latter is mainly used to store blocks, transaction indexes, and contract status.

(2) Component layer: it provides network transmission, verification mechanism, contract running engine, consensus mechanism, and other components and provides basic services for the application interface layer.
(3) Application interface layer: it provides an external interface to interact with the blockchain system in the form of RESTful API; the application interface layer provides basic functions such as transaction submission, and transaction and block retrieval.

(4) Application layer: in combination with the business process of medical waste supervision, the management of contracts, interfaces, and digital credentials is realized based on the application interface layer from the four aspects of collection, storage, transportation, and disposal.

4.2. Stakeholder Alliance and Consensus Node Construction. In the deployment of blockchain, the autonomous and controllable blockchain basic component RepChain is selected as the underlying framework. RepChain is an alliance chain implemented by responsive programming, which has a good foundation in theory and engineering. Figure 7 shows the construction scheme of stakeholder alliance and consensus node.

(1) RepChain is used as the evidence chain of medical waste data, and medical institutions, transportation departments, transportation companies, environmental protection departments, disposal companies, and other subjects are used as alliance nodes, whose cores are various devices with the capabilities of computing, data storage, and telecommunication. Regulatory agencies and operators can participate in the RepChain as a node using these connected 


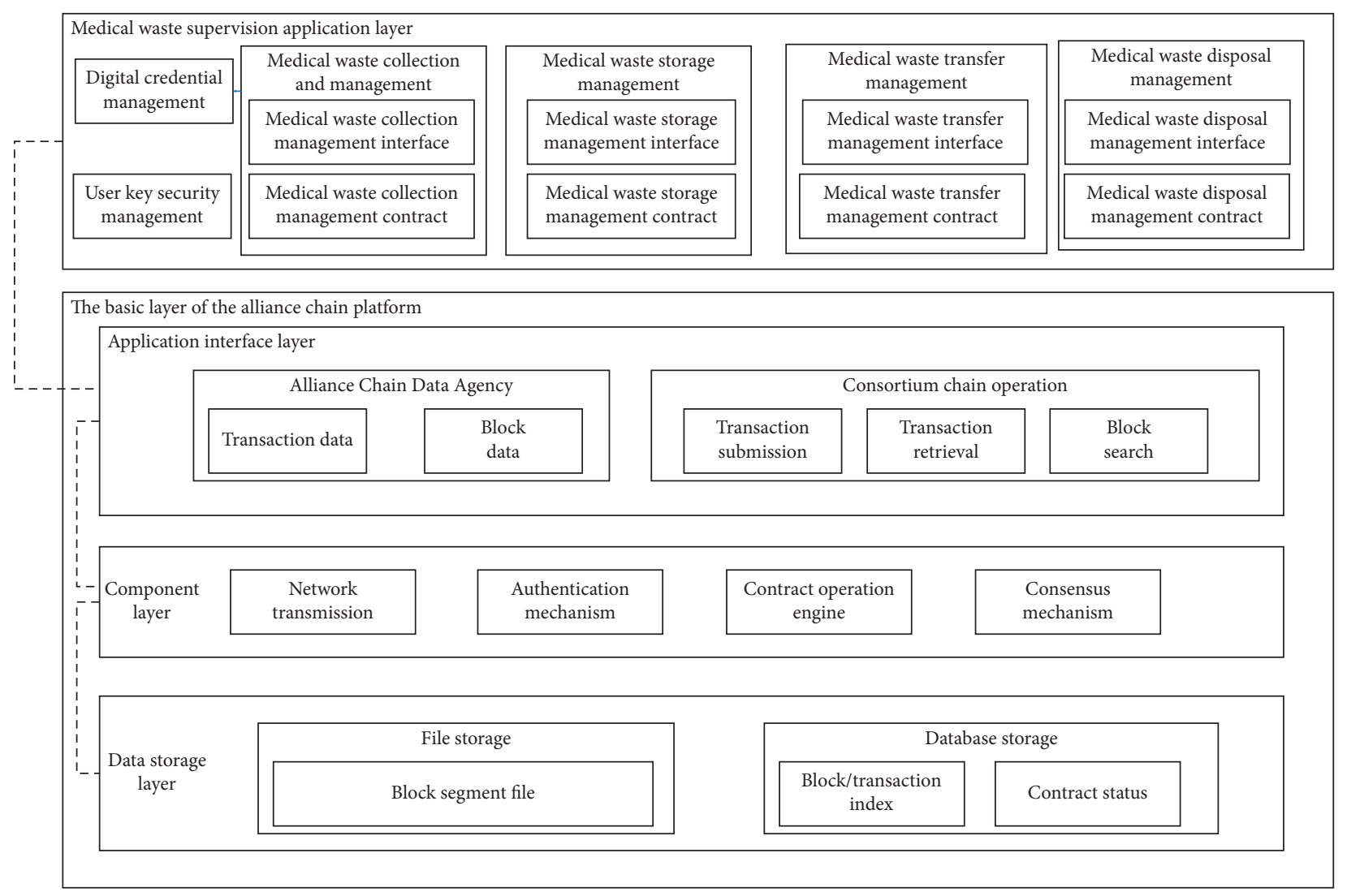

Figure 6: Regulatory framework for medical waste.

devices, and the critical data of key links are linked in the whole process to ensure the authenticity and integrity of data.

(2) Smart contracts are used to store and retrieve medical waste and submit it to the blockchain in the form of signature transactions to ensure that all participants have clear rights and responsibilities.

(3) The block information is viewed according to the visual real-time state diagram provided by RepChain. Any institution or user who can access the blockchain data can view the data on the chain and verify the authenticity of the information on the chain.

4.3. Deployment and Implementation of Medical Waste Supervision System. The deployment architecture of the medical waste supervision system is composed of a front exchange area, Web layer, application server layer, database server layer, and storage area. Our goal is to establish a service with quick, reliable, and sustainable responses. The structure is shown in Figure 8.

4.3.1. Front Exchange Layer. By deploying a set of front-end processors, it can receive data from outside, develop a unified interface and data standards, realize the function of data exchange, receive and send data files in real time or regularly, and separate the data exchange module from other functional modules of the platform, which reduces the risk of operation and improves the reliability of the system.

4.3.2. Web Layer. According to the use of resources, the information request operation is forwarded, and the user's access platform is judged and switched according to the user's request. When Web browser connects to a server and requests a file, the server processes the request and sends the file to the Web browser, along with information that tells the browser how to view the file. The server uses the HTTPS protocol for information exchange to ensure the security of information transmission.

4.3.3. Application Server Layer. It mainly deploys the application program supporting the business implementation, specifically processes the request transmitted by the Web layer, and returns the corresponding processing results according to the corresponding business logic. The application server uses load balancing technology on multiple servers to bear the access pressure of the whole system according to certain rules. Each server has the same status, which can handle the high concurrent requests that one server cannot bear at the same time and cannot affect the operation of the whole system when one server fails.

4.3.4. Database Server Layer. It is mainly used to process data query or data manipulation requests, and the 


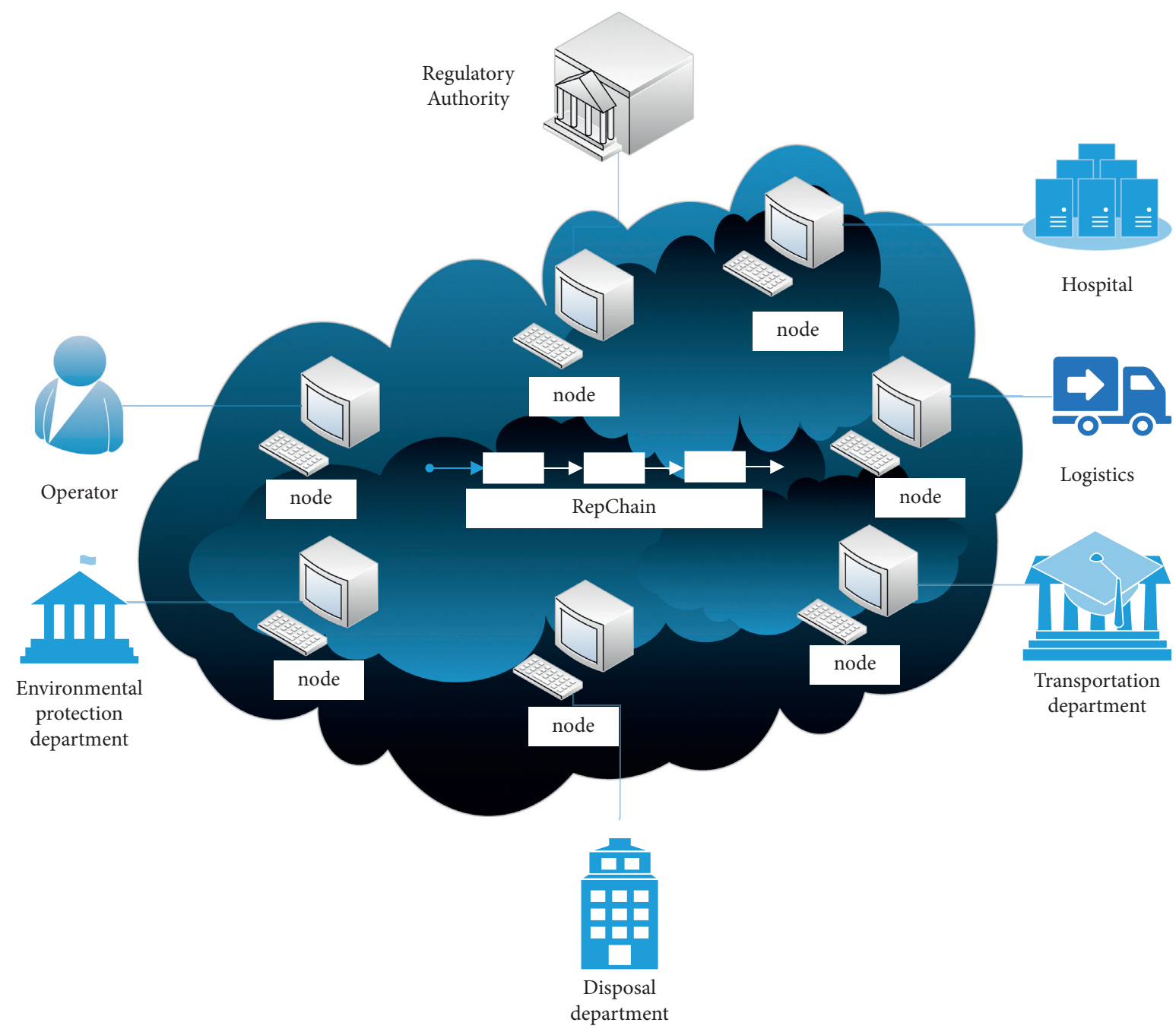

FIgURE 7: Stakeholder alliance and consensus node construction scheme.

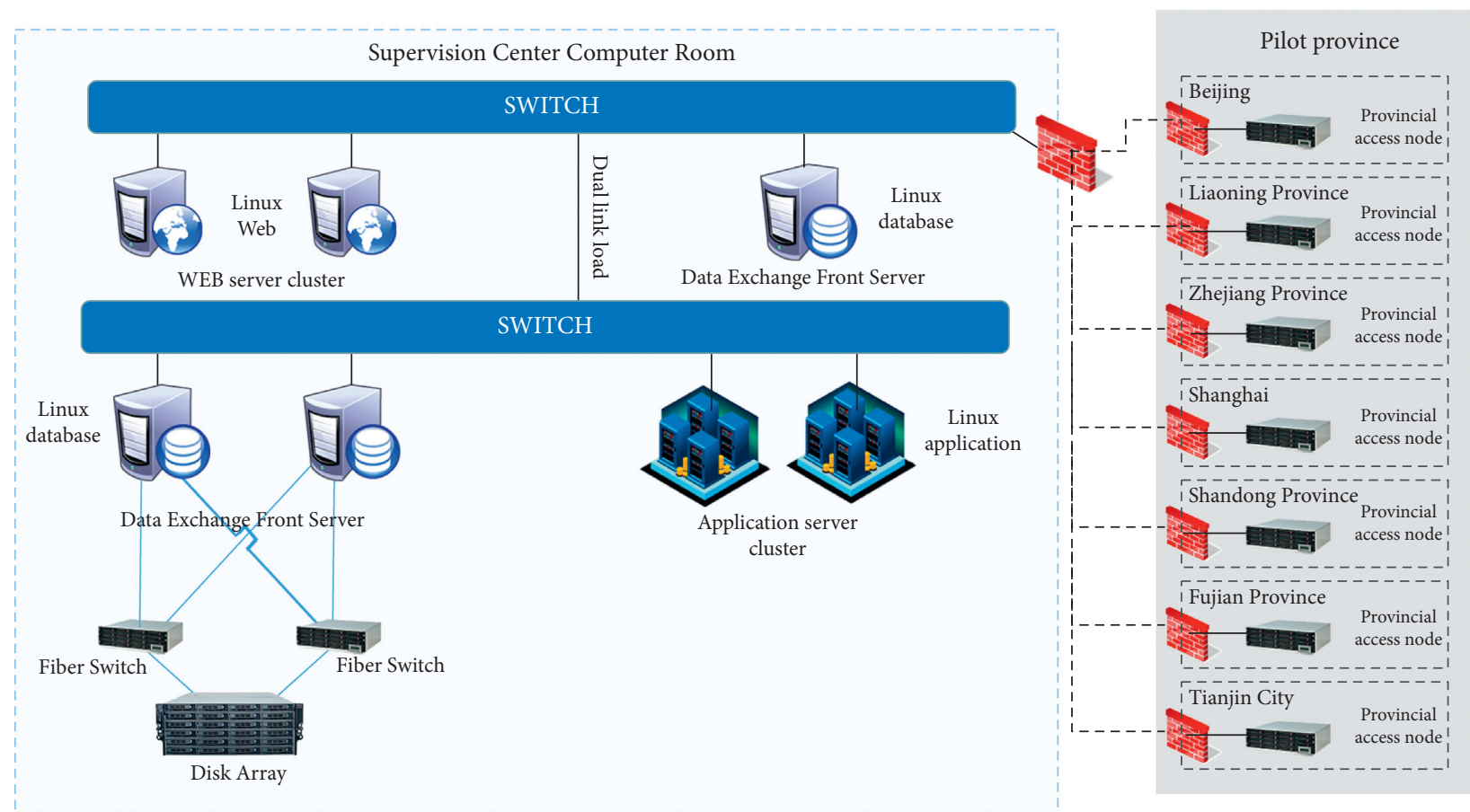

FIGURE 8: Deployment structure of medical waste supervision system. 
application part that interacts with the user runs on the user's workstation. At the same time, transaction management, indexing, caching, query optimization, security, and multiuser access control are implemented.

4.3.5. Storage Area. It is mainly composed of a fiber switch and a disk array. The disk array comprises many cheaper disks, which are combined into a disk group with huge capacity. The performance of the whole disk system is improved by using the additive effect of the data provided by individual disks.

The format of medical waste data is designed, including fields such as name, type, and description. See Table 1 for details.

Uplink data is divided into two major categories: data oriented to account permissions and data oriented to business. The chaining data for account authority mainly includes the digital certificates of each participant for account authority management, wherein each participant can use the account management contract to establish an account for a natural person in charge of specific business activities as needed, and the digital certificates are linked in the same form.

Business-oriented uplink data can be divided into two categories: basic information data and full-process traceability information data. These two parts of information collected by nodes clarify the responsibility and content of each step and make further consolidation and arrangement possible. Among them, the basic information data mainly refers to the data of relative forms unrelated to the transfer and treatment of medical waste, including but not limited to the following:

(i) Basic information on medical waste

(ii) Basic information of the medical institution

(iii) Basic information of the department

(iv) Information of medical waste temporary storage point

(v) Information on medical waste transfer vehicles

(vi) Medical waste destruction point information

(vii) Information on various types of personnel The information of the input participant is linked to its digital certificate and identity. The whole-process traceability information data mainly refers to the dynamically increased data generated from the generation, classification, packaging, temporary storage, in-hospital transfer, out-of-hospital transfer, and final disposal of medical waste, including but not limited to the following:

(i) Packaging traceability code

(ii) Medical waste classification label

(iii) Weighing information

(iv) Documentary photos

(v) Handover information

(vi) Trajectory information during transportation (vii) Surveillance video evidence information

In the chaining process, the data related to a single participant needs to be signed by the participant with the private key corresponding to its digital certificate to ensure that the participant has an undeniable responsibility for the data. The data generated by the handover activity involving multiparty participation needs to be signed by the private key corresponding to the digital certificate of the multiparty participants and then can be linked to ensure that the participants agree that the data is authentic and credible. It is also necessary to establish the association relationship between the data records through unique identification and reference for the uplink information. In addition, each link forms a closed loop by recording the identifier of the preceding step in the chain-up data generated by the handover step, which means the procedures are well connected at an information level. The critical data can be stored in the latest chain-up state through a set structure such as an array, so that the state can be quickly viewed.

\section{Demonstration of Medical Waste Supervision Model Based on Blockchain}

The IoT-based and blockchain-based medical waste supervision model proposed in former sections has been employed in the industry and shown the capacity for efficient and reliable digitalized management of waste products, which is a valid proof of the feasibility of our system.

5.1. Practical Application of the Model. The research and development of the medical waste monitoring system were completed in 2018, and the system was put into operation in pilot hospitals in Shaanxi Province in June 2020. See Tables 2 and 3 for the cumulative monitoring of medical waste disposal. Table 2 presents the statistics of medical waste categories, namely, infectious waste, loss waste, pharmacological waste, chemical waste, and pathological waste; Table 3 shows the statistics of handover links, namely, department handover, nursing handover, warehouse management handover, and transportation handover.

The medical waste smart contract deposit algorithm is shown in Figure 9, and the traceability algorithm is shown in Figure 10.

Figure 9 introduces the storage certificate algorithm. The blockchain-based medical waste disposal system uses the storage certificate smart contract to upload medical wasterelated information to the blockchain, which mainly includes basic medical waste information, basic information of medical institutions, basic information of departments, and medical treatment. The information is mainly collected from temporary waste storage sites, medical waste transfer vehicles, medical waste destruction sites, various personnel information, and so on. Each user has an independent private key to ensure that only users or institutions permitted by the blockchain can initiate requests. If an unregistered user or institution initiates the request, it will be directly rejected. Regarding the data on the chain, a hash function is used to encrypt the string to generate a unique, conflict-free, and 
TABLE 1: Categories of medical wastes.

\begin{tabular}{|c|c|c|c|}
\hline Name & Type & Description & Remark \\
\hline PackageId & String & Waste bag number & The smallest unit of waste \\
\hline BoxId & String & Shipping box number & Packing the waste and packing it into a box \\
\hline VehicleId & String & Transport vehicle number & Loading waste after packing \\
\hline MW_Type & String & Waste category & Plaintext \\
\hline MW_Weight & String & Waste weight & \\
\hline Op_Role & String & On-chain role category & $\begin{array}{l}\text { (1) Hospital } \\
\text { (2) Transportation } \\
\text { (3) Disposal }\end{array}$ \\
\hline Op_UnitId & String & Operator unit ID & \\
\hline Op_MemberId & String & Operator ID & \\
\hline Op_MemberDigest & String & Operator information digest hash & \\
\hline Operation & String & Operational behavior & $\begin{array}{l}\text { (1) Initial packaging/confirmation } \\
\text { (2) Packing/confirmation } \\
\text { (3) Loading/confirmation } \\
\text { (4) Temporary storage/confirmation of warehousing } \\
\text { (5) Recycling/confirmation }\end{array}$ \\
\hline Oth_MemberId & String & Handover ID & \\
\hline Op_DateTime & String & Operating time & Timestamp \\
\hline Op_Location & String & Operating location coordinates & Traditional latitude and longitude format \\
\hline Op_RFIDInfo & String & RFID location coordinate information & \\
\hline Memo_Photo & String & Photo digest hash & \\
\hline
\end{tabular}

Note. The above table only shows the design of some uplink data formats.

TABle 2: Statistics of categories of medical wastes.

\begin{tabular}{lc}
\hline Medical waste category & Weight $(\mathrm{kg})$ \\
\hline Infectious waste & 1467.84 \\
Lossy waste & 105.58 \\
Pharmacological waste & 81.32 \\
Chemical waste & 43.05 \\
Pathological waste & 113.68 \\
\hline
\end{tabular}

TABLE 3: Statistics of handover link.

\begin{tabular}{lc}
\hline Handover & Quantity (article) \\
\hline Department handover & 889 \\
Nurse handover & 88 \\
Library management handover & 54 \\
Transport handover & 49 \\
\hline
\end{tabular}

irreversible identifier. The PROOF function is used to store medical waste-related information on the blockchain. Before that, it will determine whether there is duplicate data on the blockchain. If there is, it will show that it has been on the chain. If not, it will be stored on the blockchain.

Figure 10 introduces the traceability algorithm. The retrieval function user checks whether the comparison chain is consistent between the upper and lower chains. After the medical waste-related data is stored in the blockchain, when the verifier wants to verify whether the data is true and reliable, on the one hand, it can pass any section. The three-party tool performs verification, submission, and production of electronic evidence document summaries. On the other hand, it can obtain the deposit transaction from the block of the blockchain and obtain the data set from it, so that the two can be compared and the verification result can be obtained from it.
5.2. Application Effect Demonstration. The medical waste supervision system saves the tedious manual recording and data analysis for medical institutions and realizes the excellent management of medical waste by collecting required information and facilitating data exchange. Similarly, only smartphones need to be used for health supervision agencies to trace back and monitor the whole process of medical waste disposal in real time, around the clock. The medical waste supervision system interface is shown in Figure 11, and Figure 12 shows the signature and verification of the blockchain transaction data.

Through the innovative blockchain application and the IoT, the medical waste supervision system promotes the standardized and digitalized management of medical waste. Through online real-time monitoring, whole-process monitoring, and other informatized means, it focuses on solving the difficulties of medical waste supervision in health supervision and improves the informatization and intelligence level of health supervision.

5.3. Related Research. The studies listed in Table 4 are more relevant to this article. Compared with these works, the advancement of this work is reflected in privacy protection. We propose a verifiable credential implementation method based on atomic signature. The method includes the following: (a) a credential structure creator defines and creates a specific verifiable credential structure with various properties. (b) Based on the atomic signature mechanism, the credential issuer constructs a verifiable credential complying with the above verifiable credential structure. (c) The credential holder checks the validity of the verifiable credential. (d) The credential holder selectively discloses and presents the specific attributes information with the 


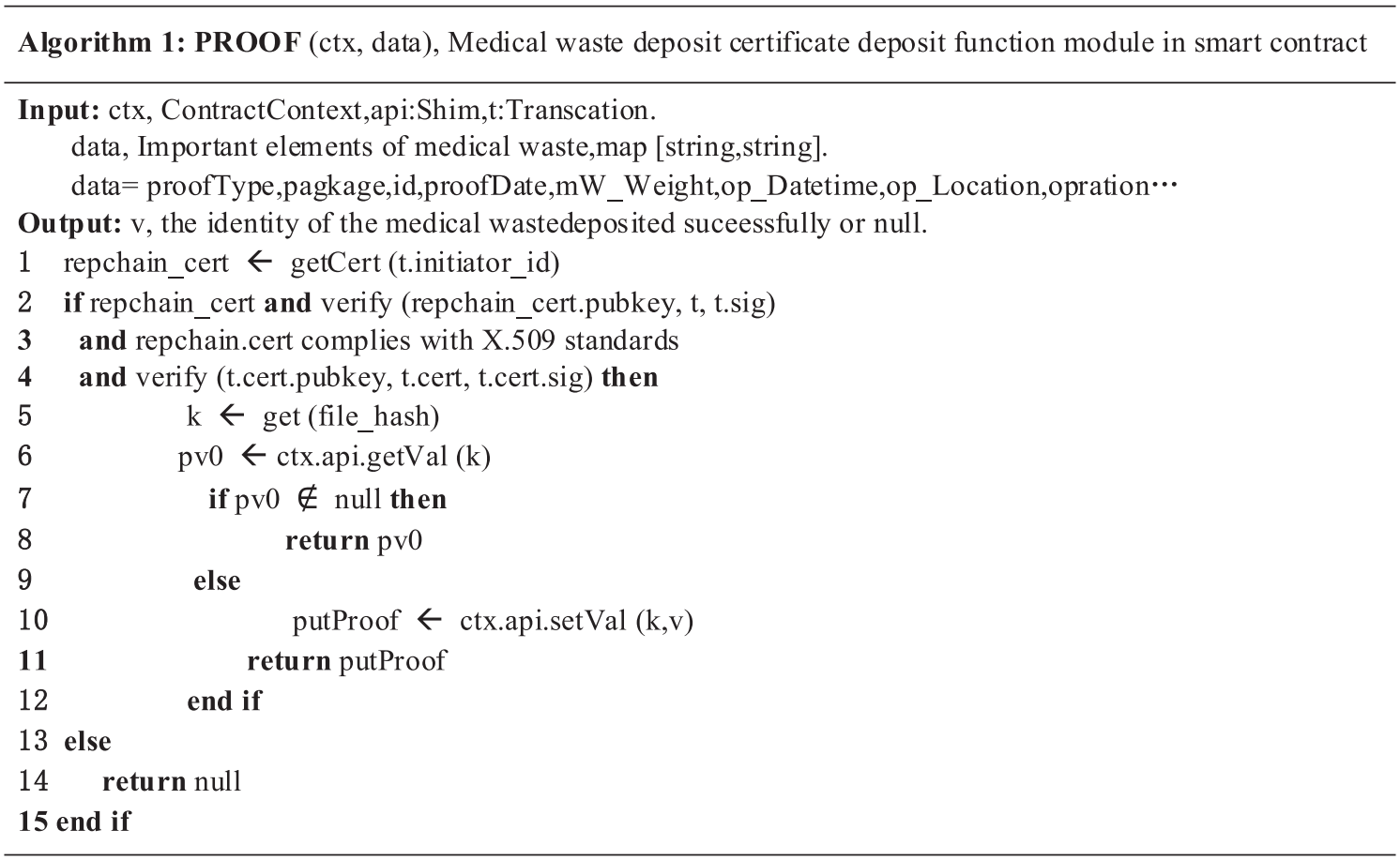

FIGURE 9: Medical waste smart contract certificate algorithm.

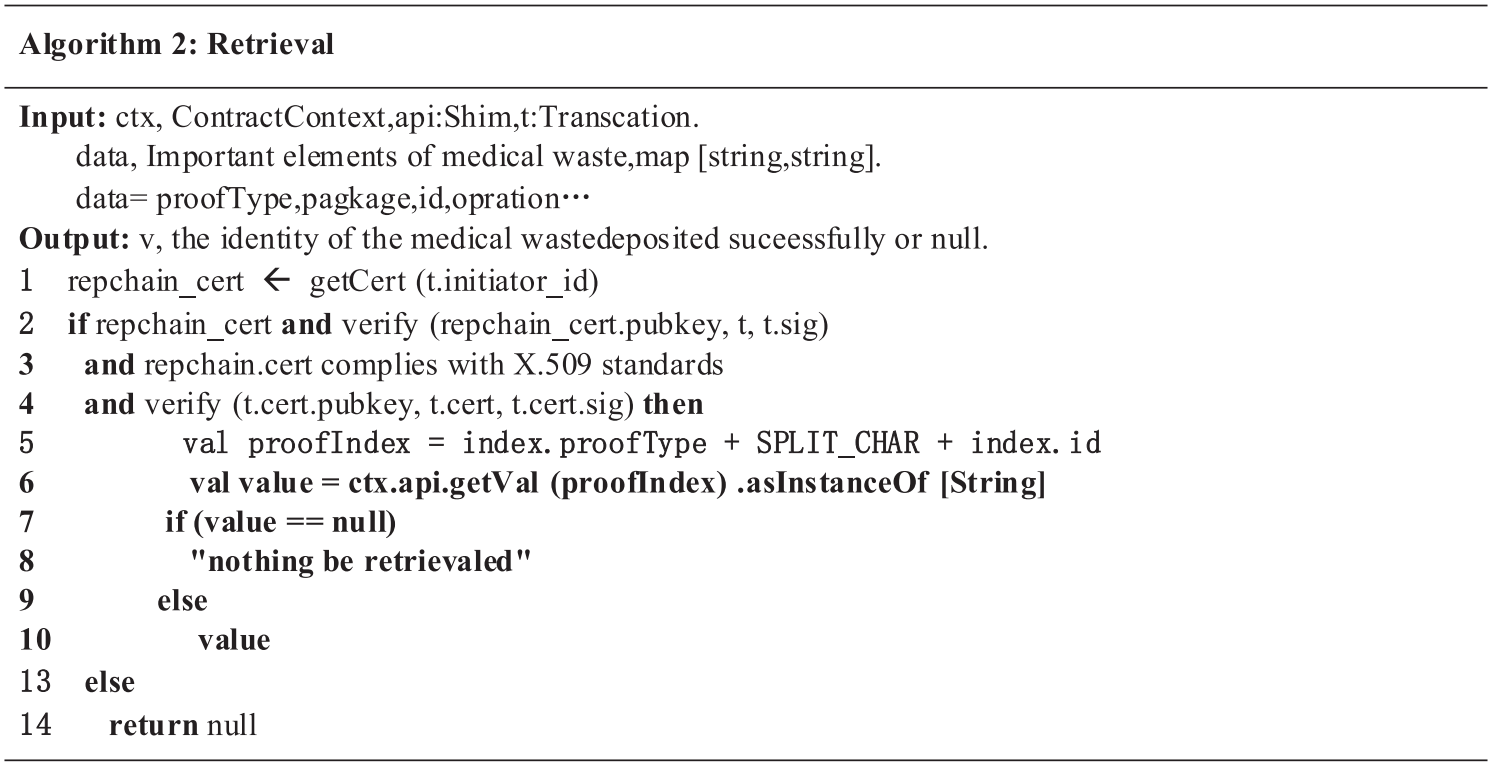

FIGURE 10: Intelligent contract traceability algorithm for medical waste.

respective atomic signatures to a credential verifier. (e) When receiving the presentation, the credential verifier verifies its authenticity and validity. (f) The credential issuer could either entirely update the whole verifiable credential or partly update some attributes of the verifiable credential.

5.4. Case Study. This work has been successfully applied in Shaanxi Province, China, in 2020, and because of certain restrictions, we cannot get specific data. Therefore, we have added a case study to the article and conducted some analysis on the reasons for the successful application of this work in Shaanxi Province.

In 2019, the National Health Commission of China randomly inspected 41,337 medical and health institutions for medical waste and imposed administrative penalties on 2,122 institutions that violated the relevant regulations on medical waste disposal, accounting for $80 \%$ of the number of investigations and punishments of medical and health institutions for violations of infectious disease prevention and control. 


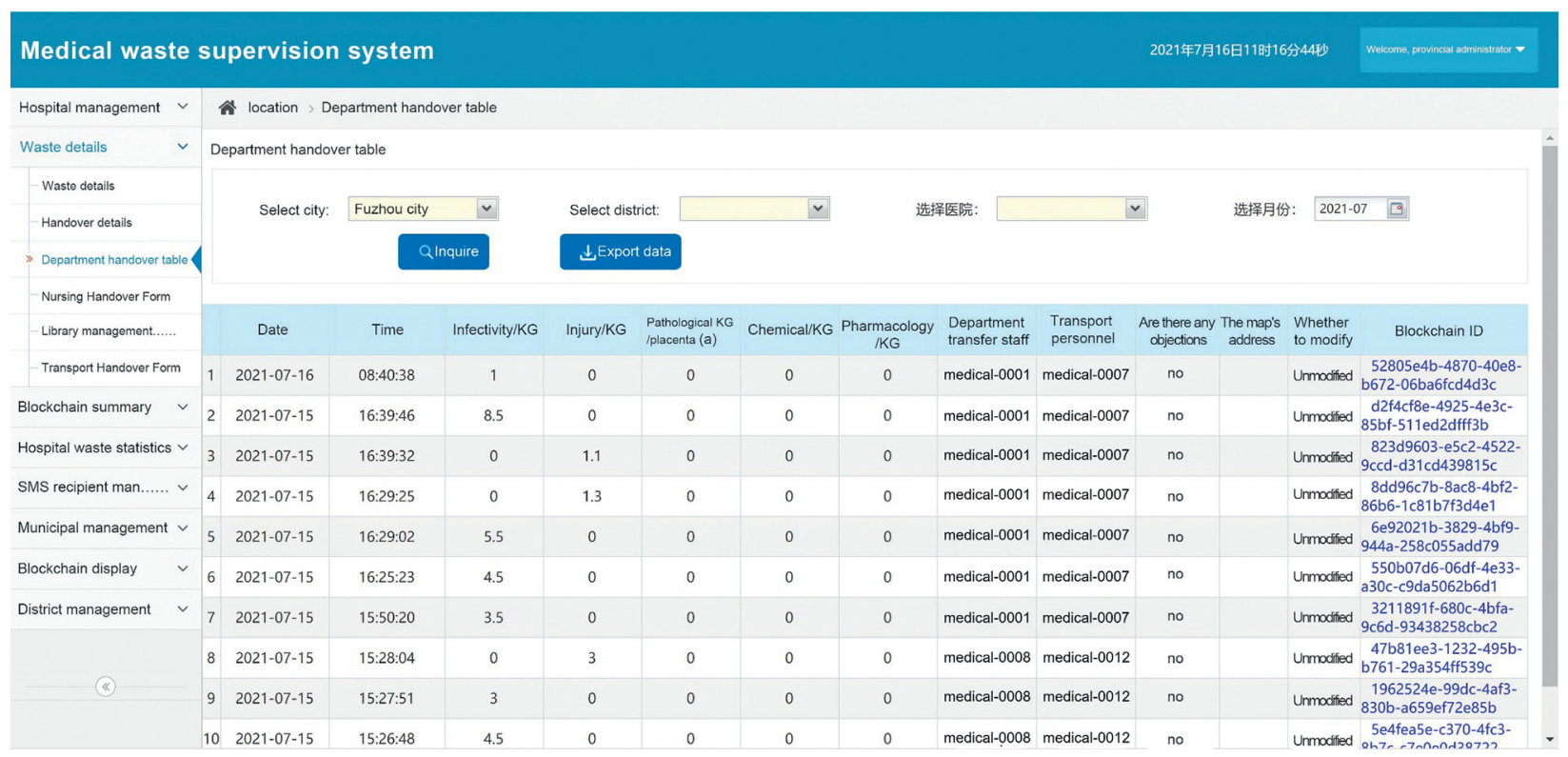

Figure 11: Interface of medical waste supervision system.

\begin{tabular}{|c|c|c|c|c|c|c|c|}
\hline & \multirow{2}{*}{\multicolumn{6}{|c|}{ Blockchain verification }} & \\
\hline & & & & & & & \\
\hline & \multicolumn{6}{|c|}{ Transaction ID: f40ad6d0-5e53-4e50-960e-128a899e44ac } & \\
\hline & \multicolumn{6}{|c|}{$\begin{array}{c}\text { Transaction ID } \\
\text { f40ad6d0-5e53-4e50-960e-128a899e44ac }\end{array}$} & \\
\hline & \multicolumn{6}{|c|}{$\begin{array}{l}\text { Transaction time } \\
\text { 2019-04-22T07:11:02.977Z }\end{array}$} & \\
\hline & \multicolumn{6}{|c|}{$\begin{array}{l}\text { Transaction Type } \\
\text { wasteProof }\end{array}$} & \\
\hline & \multicolumn{6}{|c|}{ Contract ID } & \\
\hline & \multicolumn{6}{|c|}{$\begin{array}{c}\text { cGF0aDogInBhdGgiCm5hbWU6ICIxODIONGFmODAxNzdkODNmOTMzNjFhNjAxMGZIZjA1ZmQ1NZAyZWRI } \\
\text { ZIgONmZiYjEwMDQxMzZjYjA5MTU1YjhjIgo }=\end{array}$} & \\
\hline & \multirow{2}{*}{\multicolumn{6}{|c|}{$\begin{array}{c}\text { Transaction certificate } \\
\text { MUx1djV2cTR2MUNSa1ROOThZTWhxUVYxRjE4bkd2MTFnWA== }\end{array}$}} & \\
\hline & & & & & & & \\
\hline & \multicolumn{6}{|c|}{$\begin{array}{c}\text { Transaction signature } \\
\text { MEUCIQCMW/2k25bhDdjAqrdmS5KXmPosGa0qmV9YKU6ODFRpfwIgFuvZkgnOGNZ4wsyhV8ByWNPBLum } \\
\text { EKvbPa8UHPYNvqZU }=\end{array}$} & \\
\hline & \multicolumn{6}{|c|}{ 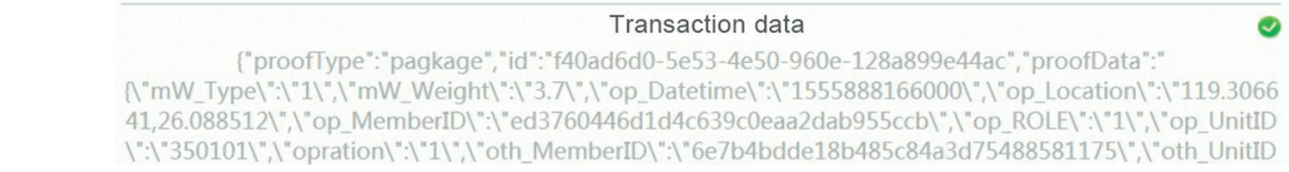 } & \\
\hline \multicolumn{8}{|c|}{ FIGURE 12: Signature and verification of blockchain transaction data. } \\
\hline Study & Waste type & Shipping rules & Traceability & Decentralized & Supply chain & Waste frauds & Privacy protection \\
\hline$[30]$ & Domestic waste & No & Yes & No & No & No & No \\
\hline [31] & Electronic waste & No & Yes & No & Forward & No & No \\
\hline$[32]$ & Domestic waste & No & No & No & No & No & No \\
\hline [33] & N/A & No & Yes & Yes & Forward & No & No \\
\hline [29] & Domestic waste & No & No & No & No & No & No \\
\hline [24] & COVID-19 MW & Yes & Yes & Yes & Forward & Yes & No \\
\hline Our study & Medical waste & Yes & Yes & Yes & Forward & Yes & Yes \\
\hline
\end{tabular}


In recent years, the administrative departments of health and environmental protection, through deepening the reform of the medical and health system, are focusing on solving the difficulties and pain points in managing medical waste disposal in small and medium medical institutions. Examples include (a) the integrated management of rural medical and health institutions; (b) the functions of new medical management mechanisms such as the medical community; (c) exploring the management model of centralized medical waste from primary medical and health institutions to the higher-level medical and health institutions for unified disposal; and (d) transporting to the nearest medical waste centralized disposal unit that holds a hazardous waste business license, namely, suitable disposal.

This work explores and promotes the implementation of the "blockchain + medical waste supervision" model in Shaanxi Province, China. We use blockchain technology to digitally monitor the entire process of medical waste generation, storage, and transfer in medical and health institutions. The traces of medical waste can be detected throughout the entire process. Through big data analysis, we can grasp the actual production and centralized disposal weight of various types of medical waste and respond to abnormal situations in a timely manner.

In May 2021, at the National Medical Waste Management Work Conference held in Shaanxi Province, the Shaanxi Provincial Health Commission summarized and promoted the "blockchain + medical waste supervision" model to all provinces. In this way, the role of blockchain technology in supervision during and after the event has been fully brought into play. Blockchain technology promotes regulatory innovation, promotes the modernization of medical waste management capabilities, and realizes the maximization of regulatory efficiency, the optimization of regulatory costs, and the minimization of human interference.

\section{Conclusion}

This paper proposes a medical waste supervision model based on blockchain, which combines blockchain technology with digital evidence provided by directly involved individuals or institutions. On the one hand, it can provide an efficient and transparent way of supervision, ensure the authenticity and integrity of data, improve the medical waste supervision system, and enhance the credibility of regulations. On the other hand, the detailed information of personnel is encapsulated in digital credentials, which effectively solves the user privacy problem existing in the traditional medical waste supervision processes and can effectively promote the healthy development of the medical waste supervision industry. The decentralized IoT model for medical waste supervision proposed in this paper provides a new idea for the transformation and upgrading of existing medical waste supervision models and implementation methods. Furthermore, the model shows the capability and importance of IoT-based blockchain in integrating and managing disperse information which can bring tangible and revolutionary changes to various areas.
The possible future directions of this work can be the application of the proposed model in different scenarios. In addition, other algorithms and technologies can be considered to further improve the performance and portability of the system.

\section{Data Availability}

All the data are collected in the medical waste monitoring system since our paper focuses on building this system rather than investing in the data. If anyone pursues further investigation of the available data, please contact the corresponding author. Moreover, we will help in extracting the data from the real-world system.

\section{Conflicts of Interest}

The authors declare that they have no conflicts of interest.

\section{References}

[1] M. Kassou, S. Bourekkadi, S. Khoulji, K. Slimani, H. Chikri, and M. L. Kerkeb, "Blockchain-based medical and water waste management conception," in Proceedings of the International Conference on Innovation, Modern Applied Science \& Environmental Studies (ICIES2020), Kenitra, Morraco, February 2021.

[2] A. S. L. França, J. A. Neto, R. F. Gonçalves, and C. M. V. B. Almeida, "Proposing the use of blockchain to improve the solid waste management in small municipalities," Journal of Cleaner Production, vol. 244, Article ID 118529, 2020.

[3] A. Kalla, T. Hewa, R. A. Mishra, M. Ylianttila, and M. Liyanage, "The role of blockchain to fight against COVID19," IEEE Engineering Management Review, vol. 48, no. 3, pp. 85-96, 2020.

[4] A. A. Abd-Alrazaq, M. Alajlani, and D. Alhuwail, "Blockchain technologies to mitigate COVID-19 challenges: a scoping review," Computer Methods and Programs in Biomedicine Update, vol. 1, Article ID 100001, 2020.

[5] M. Kouhizadeh, S. Saberi, and J. Sarkis, "Blockchain technology and the sustainable supply chain: theoretically exploring adoption barriers," International Journal of Production Economics, vol. 231, Article ID 107831, 2021.

[6] Z. Zheng, S. Xie, H. N. Dai, X. Chen, and H. Wang, "Blockchain challenges and opportunities: a survey," International Journal of Web and Grid Services, vol. 14, no. 4, pp. 352-375, 2018.

[7] D. Berdik, S. Otoum, N. Schmidt, D. Porter, and Y. Jararweh, "A survey on blockchain for information systems management and security," Information Processing \& Management, vol. 58, no. 1, Article ID 102397, 2021.

[8] S. Tanwar, K. Parekh, and R. Evans, "Blockchain-based electronic healthcare record system for healthcare 4.0 applications," Journal of Information Security and Applications, vol. 50, Article ID 102407, 2020.

[9] H. Feng, X. Wang, Y. Duan, J. Zhang, and X. Zhang, “Applying blockchain technology to improve agri-food traceability: a review of development methods, benefits and challenges," Journal of Cleaner Production, vol. 260, Article ID 121031, 2020.

[10] L. Tseng, L. Wong, S. Otoum, M. Aloqaily, and J. B. Othman, "Blockchain for managing heterogeneous internet of Things: a 
perspective architecture," IEEE Network, vol. 34, no. 1, pp. 16-23, 2020.

[11] R. W. Ahmad, K. Salah, R. Jayaraman, and I. Yaqoop, "Blockchain-based forward supply chain and waste management for COVID-19 medical equipment and supplies," IEEE Access, vol. 9, pp. 44905-44927, 2021.

[12] X. Li, P. Jiang, T. Chen, X. Luo, and Q. Wen, "A survey on the security of blockchain systems," Future Generation Computer Systems, vol. 107, pp. 841-853, 2020.

[13] F. Jamil, S. Ahmad, N. Iqbal, and D.-H. Kim, "Towards a remote monitoring of patient vital signs based on IoT-based blockchain integrity management platforms in smart hospitals," Sensors, vol. 20, no. 8, 2195 pages, 2020.

[14] P. Singh and N. Singh, "Blockchain with IoT and AI," International Journal of Applied Evolutionary Computation, vol. 11, no. 4, pp. 13-27, 2020.

[15] S. B. Rane and S. V. Thakker, "Green procurement process model based on blockchain-IoT integrated architecture for a sustainable business," Management of Environmental Quality: International Journal, vol. 31, no. 3, pp. 741-763, 2019.

[16] S. E. Chang, Y.-C. Chen, and M.-F. Lu, "Supply chain reengineering using blockchain technology: a case of smart contract based tracking process," Technological Forecasting and Social Change, vol. 144, pp. 1-11, 2019.

[17] C. R. Bass, B. Benefield, D. Horn, and R. Morones, "Increasing robustness in long text classifications using background corpus knowledge for token selection," SMU Data Science Review, vol. 2, no. 3, 10 pages, 2019.

[18] C. X. Li, S. Chen, L. S. Zheng, C. Zuo, B. Y. Jiang, and G. Liang, "RepChain-a permissioned blockchain toolkit implemented by reactive programming," Journal of Software, vol. 30, no. 6, pp. 1670-1680, 2019.

[19] Q. Xia, W. S. Dou, K. W. Guo, G. Liang, C. Zuo, and F. J. Zhang, "Survey on blockchain consensus protocol," Journal of Software, vol. 32, no. 2, pp. 277-299, 2021.

[20] N. Singh, Y. Tang, Z. Zhang, and C. Zheng, "COVID-19 waste management: effective and successful measures in Wuhan, China," Resources, Conservation and Recycling, vol. 163, Article ID 105071, 2020.

[21] H. Liu and Z. Yao, "Research on the reverse logistics management of medical waste based on the RFID technology," Fresenius Environmental Bulletin, vol. 26, pp. 8084-8092, 2017.

[22] K. Wang and W. Nai, "Application of 5G wireless communication technology in hazardous medical waste treatment," in Proceedings of the 2021 IEEE international conference on software engineering and artificial intelligence (SEAI), pp. 87-90, IEEE, Xiamen, China, June 2021.

[23] S. Li, Y. Lin, R. Ma, and Q. Chen, "Research on the integrated development of "blockchain + medical waste treatment"," Technology and Innovation, vol. 4, no. 17, pp. 20-21+24, 2019.

[24] R. W. Ahmad, K. Salah, R. Jayaraman, I. Yaqoob, M. Omar, and S. Ellahham, "Blockchain-based forward supply chain and waste management for COVID-19 medical equipment and supplies," IEEE Access, vol. 9, pp. 44905-44927, 2021.

[25] X. Jiang and Q. Tian, A Blockchain-Based Medical Waste Supervision Platform and Management Method, CN112259203A, Beijing, China, 2020.

[26] Z. Lin, On-site Treatment and Supervision System of Medical Waste Based on Internet of Things and blockchain, CN112990494A, Beijing, China, 2021.

[27] N. Gupta and P. Bedi, "E-waste management using blockchain-based smart contracts," in Proceedings of the 2018 International Conference on Advances in Computing,
Communications and Informatics (ICACCI), Chengdu, China, June 2018.

[28] M. R. Laouar, Z. T. Hamad, and S. Eom, "Towards blockchainbased urban planning: application for waste collection management," in Proceedings of the 9th International Conference on Information Systems and Technologies (icist 2019), Association for Computing Machinery, New York, NY, USA, March 2019.

[29] J. Stark, "Product lifecycle management," Product Lifecycle Management, vol. 2, pp. 1-35, 2016.

[30] M. Lamichhane, "A smart waste management system using IoT and blockchain technology," Master's Thesis in PERCCOM Master Program, ITMO University, Saint Petersburg, Russia, 2017.

[31] N. Gupta and P. Bedi, "E-waste management using blockchain based smart contracts," in Proceedings of the International Conference Advanced Computer Communication Information (ICACCI), pp. 915-921, Bangalore, India, September 2018.

[32] G. K. Shyam, S. S. Manvi, and P. Bharti, "Smart waste management using Internet-of-Things (IoT)," in Proceedings of the 2nd International Conference Computer Communication Technology (ICCCT), pp. 199-203, Chennai, India, February 2017.

[33] I. Omar, M. Debe, R. Jayaraman, K. Salah, M. Omar, and J. Arshad, "Blockchain-based supply chain traceability for COVID-19 PPE," Computers \& Industrial Engineering, vol. 167, Article ID 107995, 2022. 